\begin{tabular}{lll} 
C O L L O Q U I U M & M A T H E M A T I C U M \\
\hline vol. LXVI & 1993 & FASC. 1 \\
\hline
\end{tabular}

\title{
A NOTE ON A CONJECTURE OF D. OBERLIN
}

BY

\section{YIBIAO PAN (PITTSBURGH, PENNSYLVANIA)}

1. Let $N$ be a positive integer, $P_{N}$ be the set of all real-valued polynomials on $\mathbb{R}$ of degree at most $N$. In [1], D. Oberlin stated the following conjecture concerning uniform estimates for oscillatory integrals with polynomial phases:

Conjecture. Let $n, N$ be two positive integers. Then there is a constant $C(N, n)$ such that

$$
\left.\left|\int_{a}^{b} e^{i P(x)}\right| P^{(n)}(x)\right|^{1 / n+i s} d x \mid \leq C(N, n)(1+|s|)^{1 / n},
$$

for $P \in P_{N}, a<b$ and $s \in \mathbb{R}$.

For the significance of such estimates in Fourier analysis, we refer the reader to [1] and [2].

Clearly, (1.1) holds if $n \geq N$ (for $n>N$ it is trivial; for $n=N$ it follows from van der Corput's lemma). Hence we need to be concerned with $n=1, \ldots, N-1$ only. For $n=1$ or 2 , the conjecture has been proved by Oberlin ([1], Theorem 2). The purpose of this note is to prove the conjectured estimate (1.1) in the case $n=N-1$.

2. We state our result as the following theorem.

Theorem. Let $N \geq 2$ be an integer. Then there exists a constant $C(N)>0$ such that

$$
\left.\left|\int_{a}^{b} e^{i P(x)}\right| P^{(N-1)}(x)\right|^{1 /(N-1)+i s} d x \mid \leq C(N)(1+|s|)^{1 /(N-1)},
$$

for $P \in P_{N}, a<b$ and $s \in \mathbb{R}$.

First we state a simple lemma whose proof is deferred until the end of this note.

1991 Mathematics Subject Classification: Primary 42B20. 
LEMma 2.1. Let $n$ be a positive integer, $Q(x)$ be a monic polynomial with real coefficients and degree $n$. Suppose that the coefficient of the $x^{n-1}$ term in $Q(x)$ is zero. Then there are $m(m \leq n)$ disjoint intervals $J_{1}, \ldots, J_{m}$ and $r_{1}, \ldots, r_{m} \in \mathbb{R}$ such that $\bigcup_{k=1}^{m} J_{k}=\mathbb{R}$ and

$$
|Q(x)| \geq|x|\left|x-r_{k}\right|^{n-1},
$$

for $x \in J_{k}, k=1, \ldots, m$.

We shall need the following lemma which is due to van der Corput.

LEMma 2.2 ([3], p. 197). Suppose $\varphi$ and $\psi$ are smooth on $[a, b]$ and $\varphi$ is real-valued. If $\left|\varphi^{\prime}(x)\right| \geq \lambda$, and $\varphi^{\prime}$ is monotone on $[a, b]$, then

$$
\left|\int_{a}^{b} e^{i \varphi(x)} \psi(x) d x\right| \leq 4 \lambda^{-1}\left(|\psi(b)|+\int_{a}^{b}\left|\psi^{\prime}(x)\right| d x\right) .
$$

Proof of the Theorem. Let $P \in P_{N}$, and $\operatorname{deg}(P)=N$. By a change of variable $x \rightarrow c x+d$, for suitable $c$ and $d$, we may assume that $P(x)$ is of the form

$$
P(x)=x^{N}+R(x),
$$

with $\operatorname{deg}(R) \leq N-2$. To prove the Theorem, it suffices to prove that, for $a<b$ and $s \in \mathbb{R}$,

$$
\left.\left|\int_{a}^{b} e^{i P(x)}\right| x\right|^{1 /(N-1)+i s} d x \mid \leq C(N)(1+|s|)^{1 /(N-1)} .
$$

Let $n=N-1, Q(x)=P^{\prime}(x)$. We assume that $n \geq 2$ (for $n=1$ is covered by Oberlin's result). By Lemma 2.1, there are disjoint intervals $J_{1}, \ldots, J_{m}$ and $r_{1}, \ldots, r_{m} \in \mathbb{R}$ (for some $m \leq n$ ) such that $\bigcup_{k=1}^{m} J_{k}=\mathbb{R}$ and

$$
|Q(x)| \geq|x|\left|x-r_{k}\right|^{n-1},
$$

for $x \in J_{k}, k=1, \ldots, m$. To prove (2.4), we may assume that $P^{\prime \prime}(x)$ is of constant sign on $I=[a, b]$. As a further reduction, we shall consider the integral over each $I \cap J_{k} \cap(0, \infty)$ and $I \cap J_{k} \cap(-\infty, 0)$, for $k=1, \ldots, m$. Without loss of generality, we pick $k=1$, and consider the integral over $I \cap J_{1} \cap(0, \infty)$. For the sake of convenience, we still denote $I \cap J_{1} \cap(0, \infty)$ by $I$. Let $A=r_{1}$; we have

$$
\left|P^{\prime}(x)\right| \geq|x||x-A|^{n-1},
$$

for $x \in I$. There are two cases.

Case I: $A>0$. Let $\sigma>0$ such that $\sigma^{n}(A+\sigma)=1+|s|$. Then,

$$
\left.\left|\int_{I \cap[A, A+\sigma]} e^{i P(x)}\right| x\right|^{1 / n+i s} d x \mid \leq \sigma(A+\sigma)^{1 / n}=(1+|s|)^{1 / n} .
$$


On the other hand, by Lemma 2.2 , we have, for $j \geq 0$,

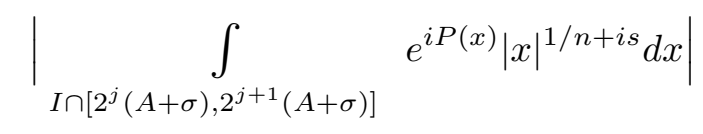

$$
\begin{aligned}
& \leq \frac{C(N)}{2^{j}(A+\sigma) \sigma^{n-1}}\left(2^{(j+1) / n}(A+\sigma)^{1 / n}+\left(\frac{1}{n}+|s|\right) \int_{2^{j}(A+\sigma)}^{2^{j+1}(A+\sigma)} x^{1 / n-1} d x\right) \\
& \leq \frac{C(N)(1+|s|)}{(A+\sigma)^{(n-1) / n} \sigma^{n-1}} 2^{(1 / n-1) j}=C(N)(1+|s|)^{1 / n} 2^{(1 / n-1) j} .
\end{aligned}
$$

Hence we have

$$
\begin{aligned}
\left.\left|\int_{I \cap[A+\sigma, \infty)} e^{i P(x)}\right| x\right|^{1 / n+i s} d x \mid & \leq C(N)(1+|s|)^{1 / n} \sum_{j \geq 0} 2^{(1 / n-1) j} \\
& \leq C(N)(1+|s|)^{1 / n}
\end{aligned}
$$

It remains for us to show that

$$
\left.\left|\int_{I \cap[0, A]} e^{i P(x)}\right| x\right|^{1 / n+i s} d x \mid \leq C(N)(1+|s|)^{1 / n} .
$$

If $A \leq 4^{n /(n+1)}(1+|s|)^{1 /(n+1)}$, then

$$
\left.\left|\int_{I \cap[0, A]} e^{i P(x)}\right| x\right|^{1 / n+i s} d x \mid \leq \int_{0}^{A} x^{1 / n} d x \leq C(N)(1+|s|)^{1 / n} .
$$

If $A>4^{n /(n+1)}(1+|s|)^{1 /(n+1)}=8 B$, we let $\sigma^{\prime}=((1+|s|) / A)^{1 / n} \leq A / 4$.

Let $n_{1}$ and $n_{2}$ be two integers such that

$$
2^{n_{1}} \leq B<2^{n_{1}+1} \quad \text { and } \quad 2^{n_{2}} \leq A / 4<2^{n_{2}+1} .
$$

We write

$$
\begin{aligned}
& \int_{I \cap[0, A]} e^{i P(x)}|x|^{1 / n+i s} d x \\
= & \int_{I \cap\left[0,2^{n_{1}}\right]} e^{i P(x)}|x|^{1 / n+i s} d x+\sum_{j=n_{1}}^{n_{2}} \int_{I \cap\left[2^{j}, 2^{j+1}\right]} e^{i P(x)}|x|^{1 / n+i s} d x \\
& +\int_{I \cap\left[2^{n_{2}+1}, A-\sigma^{\prime}\right]} e^{i P(x)}|x|^{1 / n+i s} d x+\int_{I \cap\left[A-\sigma^{\prime}, A\right]} e^{i P(x)}|x|^{1 / n+i s} d x .
\end{aligned}
$$

The first term and fourth term are easily seen to be bounded by $(1+|s|)^{1 / n}$. For the third term, one observes that

$$
\left|P^{\prime}(x)\right| \geq|x||x-A|^{n-1} \geq(A / 4)\left(\sigma^{\prime}\right)^{n-1},
$$


for $x \in I \cap\left[2^{n_{2}+1}, A-\sigma^{\prime}\right]$, and the desired bound follows from van der Corput's lemma. To treat the second term, we use

$$
\left|P^{\prime}(x)\right| \geq|x||x-A|^{n-1} \geq\left(2^{1-n}\right) 2^{j} A^{n-1}
$$

for $x \in I \cap\left[2^{j}, 2^{j+1}\right], n_{1} \leq j \leq n_{2}$. Then

$$
\begin{aligned}
& \mid \sum_{j=n_{1}}^{n_{2}} \int_{I \cap\left[2^{j}, 2^{j+1}\right]} e^{i P(x)}|x|^{1 / n+i s} d x \mid \\
& \leq C \sum_{j=n_{1}}^{n_{2}} \frac{(1+|s|)}{2^{j} A^{n-1}} 2^{j / n} \leq C(1+|s|) A^{1-n} 2^{n_{1}(1 / n-1)} \\
& \leq C(1+|s|)^{1-\frac{n-1}{n+1}}(1+|s|)^{\frac{1}{n+1}\left(\frac{1}{n}-1\right)}=C(1+|s|)^{1 / n}
\end{aligned}
$$

The above argument shows that (2.8) holds. Combining (2.5), (2.7) and (2.8), we see that case I is proved.

C a s e II: $A \leq 0$. This case is actually easier than the previous case. Now we have $\left|P^{\prime}(x)\right| \geq|x|^{n}$ for $x \in I$. Let $\delta=(1+|s|)^{1 /(n+1)}$; we decompose the integral as

$$
\begin{aligned}
\int_{I} e^{i P(x)}|x|^{1 / n+i s} d x= & \int_{I \cap[0, \delta]} e^{i P(x)}|x|^{1 / n+i s} d x \\
& +\sum_{j=1}^{\infty} \int_{I \cap\left[2^{j} \delta, 2^{j+1} \delta\right]} e^{i P(x)}|x|^{1 / n+i s} d x .
\end{aligned}
$$

While the first term is trivially bounded by $(1+|s|)^{1 / n}$, an application of van der Corput's lemma shows that the second term is also bounded by $(1+|s|)^{1 / n}$.

The proof of the theorem is now complete.

Proof of Lemma 2.1. Let $z_{1}, \ldots, z_{n}$ be the $n$ roots of $Q(x)$, and $\Delta=\left\{z_{1}, \ldots, z_{n}\right\}$. Then we have

$$
Q(x)=\prod_{j=1}^{n}\left(x-z_{j}\right)
$$

Suppose

$$
\{\operatorname{Re} z \mid z \in \Delta\}=\left\{r_{1}, \ldots, r_{m}\right\},
$$

and $r_{1}<\ldots<r_{m}$. Define

$$
J_{1}=\left(-\infty, \frac{r_{1}+r_{2}}{2}\right], \quad J_{m}=\left(\frac{r_{m-1}+r_{m}}{2}, \infty\right),
$$


and

$$
J_{k}=\left(\frac{r_{k-1}+r_{k}}{2}, \frac{r_{k}+r_{k+1}}{2}\right], \quad \text { for } k=2, \ldots, m-1 .
$$

For $x \in I_{k}, 1 \leq k \leq m$, we find

$$
\left|x-z_{j}\right| \geq\left|x-\operatorname{Re} z_{j}\right| \geq\left|x-r_{k}\right|
$$

for all $j=1, \ldots, n$. On the other hand, we have

$$
\sum_{j=1}^{n}\left(x-z_{j}\right)=n x-\sum_{j=1}^{n} z_{j}=n x
$$

where we used the fact that the coefficient of the $x^{n-1}$ term in $Q(x)$ is zero. Hence, for every $x \in \mathbb{R}$, there is a $j_{x}, 1 \leq j_{x} \leq n$, such that

$$
\left|x-z_{j_{x}}\right| \geq|x| \text {. }
$$

(2.11) and (2.12) imply that

$$
|Q(x)| \geq|x|\left|x-r_{k}\right|^{n-1},
$$

for $x \in J_{k}, k=1, \ldots, m$.

\section{REFERENCES}

[1] D. Oberlin, Oscillatory integrals with polynomial phase, Math. Scand. 69 (1991), $145-156$.

[2] E. M. Stein, Oscillatory integrals in Fourier analysis, in: Beijing Lectures in Harmonic Analysis, Princeton Univ. Press, Princeton, 1986, 307-355.

[3] A. Zygmund, Trigonometric Series, Cambridge Univ. Press, Cambridge, 1959.

DEPARTMENT OF MATHEMATICS AND STATISTICS

UNIVERSITY OF PITTSBURGH

PITTSBURGH, PENNSYLVANIA 15260

U.S.A.

Reçu par la Rédaction le 19.10.1992 